\title{
ESTUDIO DE LA RENTABILIDAD DE UNA RENTA DE JUBILACIÓN CONTRATADA A PRIMA PURA (ÚNICA Y PERIÓDICA)
}

\section{STUDYING THE RETURN OF SINGLE OR PERIODIC RISK PREMIUM TEMPORARY LIFE ANNUITIES}

\author{
MARÍA JOSÉ PÉREZ-FRUCTUOSO*
}

ANTONIO ALEGRE ESCOLANO**

Fecha de recepción: 20 de agosto de 2019

Fecha de aceptación: 30 de agosto de 2019

Disponible en línea: 15 de septiembre de 2019

Para citar este artículo/To cite this article

Perez Fructuoso, María José \& Alegre Escolano, Antonio, Estudio de la rentabilidad de una renta de jubilación contratada a prima pura (única y periódica), 50 Rev.Ibero-Latinoam.Seguros, 209-226 (2018). https://doi.org/10.11144/

\footnotetext{
Doctora Europea en Economía; Doctora en Ciencias Económicas y Empresariales; Licenciada en Ciencias Económicas y Empresariales, Licenciada en Ciencias Actuariales y Financieras. https:// orcid.org/0000-0002-3252-1631, Profesora Titular del Área de Economía Financiera y Contabilidad, Madrid Open University (UDIMA). Contacto: mariajose.perez@udima.es

** Profesor emérito de la Universidad de Barcelona, departamento de Matemática económica, financiera y actuarial. Profesor de Matemática Actuarial. Contacto: aalegre@ub.edu
} 


\section{RESUMEN}

Este trabajo analiza la rentabilidad que puede obtener un asegurado por contratar una renta diferida temporal o vitalicia. Al estar esta operación actuarial condicionada a la probabilidad de supervivencia del asegurado, la rentabilidad de esta es aleatoria y depende de la distribución de probabilidad asociada a dicha supervivencia. Definiremos la rentabilidad esperada y a partir de la distribución de probabilidad de la variable aleatoria que denominamos beneficio del asegurado, obtendremos unos parámetros de decisión que reflejen el riesgo de la operación y su influencia en dicha rentabilidad esperada. El desarrollo teórico se realizará para primas únicas y periódicas, en ambos casos, bajo la hipótesis de prima pura. Finalmente, plantearemos un análisis empírico que muestre la aplicabilidad de los resultados obtenidos, con el objetivo de que el asegurado tome sus decisiones en ambiente aleatorio con la máxima información.

Palabras clave: distribución de probabilidad de la rentabilidad real; rentabilidad máxima; rentabilidad esperada; indicadores de riesgo basados en la distribución de probabilidad de la rentabilidad real; renta vitalicia de jubilación. 


\begin{abstract}
This paper analyzes deferred temporary annuities and deferred life annuities insured's return. With both kind of actuarial transactions being conditioned to the insured's survival probability; their respective profitability is random and depends on the insured's survival probability distribution. We will define the expected return and, basing on the probability distribution of the random variable that we call the insured's benefit, we will obtain decision parameters that reflect the risk of both kinds of operations and its influence on that expected return. Our theoretical development will be carried out for single and periodic premiums, in all cases under the hypotheses of pure premium. Finally, we will present an empirical analysis of all the cases, showing the applicability of the results obtained, with the aim that the insured may avail himself with as much information as possible to make his or her decision in a random environment.
\end{abstract}

Keywords: real return probability distribution; maximum return; expected return; real return probability distribution-based risk indicators; deferred life annuity.

\title{
SUMARIO
}

INTRODUCCIÓN - 1. CÁlCULO DE LA DISTRIBUCIÓN DE LA RENTABILIDAD REAL Y LA RENTABILIDAD ESPERADA DE UNA RENTA DE SUPERVIVENCIA DIFERIDA M Y TEMPORAL N CONTRATADA A PRIMA ÚNICA - 1.1. Renta de supervivencia diferida $\mathrm{m}$ y temporal $\mathrm{n}$ a prima pura única - 1.1.1. Cálculo de la distribución de probabilidad de la rentabilidad real - 1.1.2. Cálculo de la rentabilidad esperada - 2. CÁlCULO DE LA DISTRIBUCIÓN DE LA RENTABILIDAD REAL Y LA RENTABILIDAD ESPERADA DE UNA RENTA DE SUPERVIVENCIA DIFERIDA M Y TEMPORAL N CONTRATADA A PRIMA PERIÓDICA - 2.1. Renta de supervivencia diferida $\mathrm{m}$ y temporal $\mathrm{n}$ a prima pura periódica - 2.1.1. Cálculo de la distribución de probabilidad de la rentabilidad real - 2.1.2. Cálculo de la rentabilidad esperada-3. APLICACIÓN NUMÉRICA - CONCLUSIONES - REFERENCIAS BIBLIOGRÁFICAS.. 



\section{INTRODUCCIÓN}

Desde el año 2015, el ordenamiento jurídico español ${ }^{1}$ establece la obligación de calcular la rentabilidad esperada de los seguros de vida, cuyo objetivo es crear un instrumento de comparación entre las diferentes operaciones de seguros relacionadas con la supervivencia o el fallecimiento del asegurado que dote de transparencia al mercado asegurador y facilite las decisiones de inversión de los asegurados (Devesa, et al. 2016).

Las operaciones actuariales implicadas en esta normativa son estocásticas porque tienen una componente de riesgo relacionada con la supervivencia o el fallecimiento del asegurado. Ello supone que para calcular la rentabilidad esperada deberá utilizarse una metodología que permita estudiar su comportamiento aleatorio a través de la distribución de probabilidad de la contingencia cubierta en el contrato. Diversos autores se han ocupado de esta cuestión, siguiendo en la mayoría de los casos las indicaciones de la orden ministerial anteriormente comentada.

Para Devesa, et al. 2013, la rentabilidad financiero-actuarial-fiscal es el tipo de interés obtenido como resultado de igualar el valor actuarial de las primas con el de las indemnizaciones, considerando una ley de capitalización compuesta, una tabla de mortalidad determinada y las características comerciales de la operación. El cálculo de esta rentabilidad se realiza en dos momentos del tiempo diferentes, antes de empezar la operación y en el momento actual de la misma, lo que permite analizar los cambios que en ella se presentan como consecuencia de variaciones en los flujos, probabilidad y rentabilidades futuras. Devesa, et al. 2016, determinan la rentabilidad esperada a través de una función implícita derivada resultado de igualar el valor actual de las prestaciones esperadas con los pagos esperados de primas. El tipo de interés efectivo anual resultante depende del tipo de prima de la operación, de las tablas de mortalidad empleadas y de la estructura de gastos que aplique la entidad aseguradora. Moreno, et al. 2017 definen la rentabilidad esperada como una variable aleatoria y la calculan de dos formas alternativas; como esperanza matemática de la variable aleatoria rentabilidad de la operación

1 Orden ECC/2329/2014, de 12 de diciembre, por la que se regula el cálculo de la rentabilidad esperada de las operaciones de seguro de vida y Real Decreto 1060/2015, de 20 de noviembre, de ordenación, supervisión y solvencia de las entidades aseguradoras y reaseguradoras. 
o como tanto al que se igualan los valores actuales medios esperados de las prestaciones y las primas.

Considerando en una operación de seguros de ahorro compleja, denominada renta de supervivencia, en la que se establece el pago de renta anticipada de forma temporal o vitalicia, si el asegurado de edad actual $x$, llega con vida a cumplir la edad $x+m$, siendo $m$ el diferimiento de la operación, el objetivo del presente trabajo será desarrollar las expresiones que permitan calcular la rentabilidad esperada de la misma, y proporcionar unos índices de riesgo que midan la probabilidad de no perder con la operación y la confianza del asegurado en obtener una rentabilidad real como mínimo superior al rendimiento esperado calculado. Para ello haremos que la esperanza matemática del valor actual de las prestaciones y contraprestaciones sea la misma, esto es, igualaremos a cero la esperanza matemática del valor actual del beneficio o pérdida para el asegurado como en Pérez-Fructuoso, et al., 2018 y 2019.

El elemento básico del cálculo de la rentabilidad esperada será la distribución de probabilidad de la variable aleatoria tanto efectivo anual de rendimiento. A partir de ella obtendremos los valores de las rentabilidades en cada periodo de la operación, según se produzca o no la contingencia cubierta en el contrato, además de unos coeficientes de riesgo que informarán acerca la bondad del rendimiento esperado alcanzado a través de las probabilidades de no pérdida, y de que el rendimiento real obtenido sea superior al esperado. La rentabilidad esperada, será la que resulte de igualar a cero la esperanza matemática de la distribución de probabilidad de la variable aleatoria función valor actual financiero del beneficio del producto.

La estructura del artículo es la siguiente. La Sección 2 desarrolla de forma teórica las expresiones que permiten obtener los valores de la variable tipo de interés anual efectivo y la rentabilidad esperada de una operación de renta de supervivencia a prima única bajo el supuesto de que se contrate a prima pura. En la Sección 3 se calcula teóricamente el tipo de interés efectivo anual y la rentabilidad esperada cuando la prima es periódica y pura. La sección 4 presenta presentan los resultados obtenidos de la aplicación numérica realizada en cada uno de los casos teóricos analizados en las dos secciones previas. Finalmente, la Sección 5 expone las principales conclusiones alcanzadas con el estudio. 


\section{CÁLCULO DELA DISTRIBUCIÓN DELARENTABILIDAD REAL Y LA RENTABILIDAD ESPERADA DE UNA RENTA DE SUPERVIVENCIA DIFERIDA $M$ Y TEMPORAL $N$ CONTRATADA A PRIMA ÚNICA}

\subsection{Renta de supervivencia diferida $m$ y temporal $n$ a prima pura única}

Consideramos una operación de seguro cuya prestación es una renta de supervivencia consistente en el pago, al asegurado de edad actual $\mathrm{x}$ años, de una serie de capitales financieros siempre y cuando llegue vivo a cada uno de los momentos en que dichos capitales se hacen efectivos. Suponemos que la renta de supervivencia es diferida $m$ años, temporal $n$ años, anticipada, de términos variables $\left\{\alpha_{t}\right\}_{(t=m, m+1, \ldots, m+n-1)}$ y cuya contratación se realiza mediante el pago de una prima pura única de cuantía $\Pi$ unidades monetarias. Entonces, la ecuación de equilibrio financiero-actuarial en el origen de la operación, según la cual el valor actual actuarial de las contraprestaciones debe ser igual al valor actual actuarial de las prestaciones (Promislow, 2015), viene dada por la siguiente expresión,

$$
\Pi=\sum_{t=m}^{m+n} \alpha_{t} \cdot(1+i)^{-t} \cdot \ldots{ }_{t} p_{x}
$$

donde $i$ es el tipo de interés técnico de la operación y ${ }^{\mp}{ }^{\infty}=\frac{5^{x}}{5^{x+5}}$ la probabilidad de que una persona de $x$ años sobreviva $t$ años más, obtenida a partir del número de personas vivas a cada edad, $l_{\mathrm{x}}$.

En todo el trabajo utilizaremos la notación $\sum_{\mathrm{t}=m}^{m+n} X_{t}=X_{m}+X_{m+1}+\cdots+X_{m+n-1}$ como una suma desde el extremo inferior hasta el anterior al extremo superior. En forma de sumatorio se expresaría de la siguiente forma,

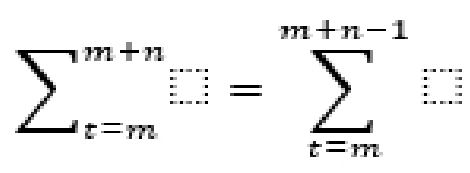




\subsubsection{Cálculo de la distribución de probabilidad de la rentabilidad real}

El tipo de interés efectivo anual de la operación, $\tilde{i}$, es una variable aleatoria cuya distribución de probabilidad viene dada por la siguiente expresión,

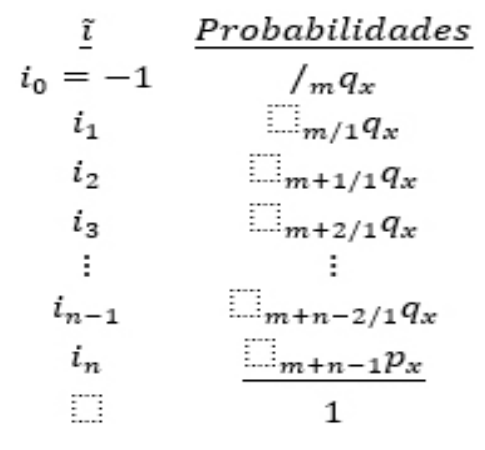

donde ${ }_{s / 1} q_{x}=\frac{l_{x+s}-l_{x+s+1}}{l_{x}} \forall s=1,2, \ldots, k, \ldots, m+n-2$ es la probabilidad de fallecimiento, diferida $\mathrm{s}$ años y temporal 1 año, cuyo cálculo se realiza a partir del número de personas vivas a cada edad, $l_{\mathrm{x}-}$.

De esta forma, si el asegurado fallece antes de que finalicen los $\mathrm{m}$ años del diferimiento, la rentabilidad de la operación es $i_{0}=-1$ puesto que pierde la prima pagada y no recibe ninguna prestación, mientras que si llega con vida a la edad $x+m$, la rentabilidad $i_{\mathrm{j}}$ con $j \in\{1,2, \ldots, n\}$, será la resultante de igualar, en cada periodo, el valor actual de las contraprestaciones y prestaciones de la operación, esto es,

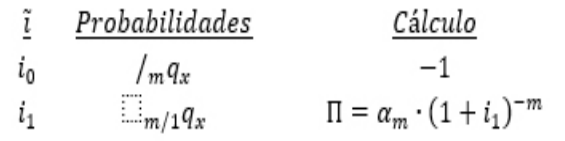

$$
\begin{aligned}
& i_{2} \quad i_{m+1 / 1} q_{x} \quad \Pi=\sum_{t=m}^{m+2} \alpha_{t} \cdot\left(1+i_{2}\right)^{-t} \\
& i_{3} \quad \Pi=\sum_{t=2 / 1}^{m+3} q_{x} \quad \cdot\left(1+i_{3}\right)^{-t} \\
& \begin{array}{ccc}
\vdots & \vdots & \vdots \\
i_{n-1} & \vdots & \vdots \\
i_{m+n-2 / 1} q_{x} & \Pi=\sum_{t=m}^{m+n-1} \alpha_{t} \cdot\left(1+i_{n-1}\right)^{-t}
\end{array} \\
& i_{n} \quad \Pi=\sum_{t=m}^{m+n} \alpha_{t} \cdot\left(1+i_{n}\right)^{-t} \\
& \text { : }
\end{aligned}
$$

donde la rentabilidad $i_{1}$ se obtiene directamente a partir de la siguiente 
expresión,

$$
\left(1+i_{1}\right)^{-m}=\frac{a_{m}}{\Pi} \Rightarrow i_{1}=\left(\frac{a_{m}}{\Pi}\right)^{\frac{1}{m}}-1
$$

con $i_{\mathrm{n}}>i$, ya que $0<{ }_{\mathrm{t}} \mathrm{p}_{\mathrm{x}}<1$.

Como indicador de riesgo relacionado con la rentabilidad real de la renta de jubilación contratada, calculamos la probabilidad $\mathrm{p}[\tilde{\mathrm{I}} \geq 0]$ que informa acerca de que la rentabilidad de la operación no sea negativa y se obtiene como la suma de todas las probabilidades diferidas de fallecimiento a partir del primer valor $i_{\mathrm{j}} \geq 0$

\subsubsection{Cálculo de la rentabilidad esperada}

Definimos la variable aleatoria función valor actual del beneficio del producto, cuya distribución de probabilidad viene dada por la siguiente expresión:

$$
\begin{array}{cc}
\tilde{E} & \text { Probabilidades } \\
-\Pi & \sum_{m} q_{x} \\
-\Pi+\alpha_{m} \cdot\left(1+i^{*}\right)^{-m} & \frac{m}{2} q_{x} \\
-\Pi+\sum_{t=m}^{m+2} \alpha_{t} \cdot\left(1+i^{*}\right)^{-t} & \frac{m+\frac{1}{2}}{2} q_{x} \\
-\Pi+\sum_{t=m}^{m+3} \alpha_{t} \cdot\left(1+i^{*}\right)^{-t} & \vdots \\
\vdots & \vdots \\
-\Pi+\sum_{t=m}^{m+n-1} \alpha_{t} \cdot\left(1+i_{n-1}\right)^{-t} & \vdots \\
-\Pi+\sum_{t=m}^{m+n} \alpha_{t} \cdot\left(1+i_{n}\right)^{-t} & \frac{2-\frac{2}{1}}{2} q_{x} \\
\vdots & 1
\end{array}
$$

La rentabilidad esperada, $i^{*}$ se obtiene haciendo $\mathbf{E}[\mathrm{B}]=\mathbf{O}$ tal que:

$$
\Pi=\sum_{j=1}^{n}\left(\sum_{t=m}^{m+j} \alpha_{t} \cdot\left(1+i^{*}\right)^{-t}\right) \cdot:_{m+j-1 / 1} q_{x}+\left(\sum_{t=m}^{m+n} \alpha_{t} \cdot\left(1+i^{*}\right)^{-t}\right) \cdot \vdots_{m+n-1} p_{x}
$$

Operando en las sumas de la expresión (6), dicha ecuación resulta: 


$$
\Pi=\sum_{t=m}^{m+n} \alpha_{t} \cdot\left(1+i^{*}\right)^{-t} \cdot \sum_{j=t-m+1}^{n} \vdots_{m+j-1 / 1} q_{x}+\left(\sum_{t=m}^{m+n} \alpha_{t} \cdot\left(1+i^{*}\right)^{-t}\right) \cdot \vdots_{m+n-1} p_{x}
$$

Entonces, considerando que,

$$
\sum_{j=t-m+1}^{n} \vdots_{m+j-1 / 1} q_{x}=\sum_{t} p_{x}-\vdots_{m+n-1} p_{x}
$$

tenemos la ecuación que permite obtener la rentabilidad esperada de la renta de supervivencia analizada, mediante un proceso iterativo:

$$
\Pi=\sum_{t=m}^{m+n} \alpha_{t} \cdot(1+i)^{-t} \cdot p_{x}
$$

Además, substituyendo П por su valor dado en la ecuación (1) se observa fácilmente que la rentabilidad esperada coincide con el tipo de interés técnico cuando la operación se contrata a prima pura única, $i^{*}=i$.

Otro indicador de riesgo, relacionado con la rentabilidad esperada, en este caso, vendrá dado por la probabilidad $p\left[\tilde{l} \geq i^{*}\right]$ que indica la confianza que tiene el asegurado en obtener una rentabilidad real que sea como mínimo la rentabilidad esperada.

\section{CÁLCULODELA DISTRIBUCIÓN DELARENTABILIDAD REAL Y LA RENTABILIDAD ESPERADA DE UNA RENTA DE SUPERVIVENCIA DIFERIDA M Y TEMPORAL N CONTRATADA A PRIMA PERIÓDICA}

\subsection{Renta de supervivencia diferida $m$ y temporal $n$ a prima pura periódica}

Consideramos una renta de supervivencia, diferida $m$ años, temporal $n$ 
años, anticipada y de términos variables $\left\{\alpha_{\mathrm{t}}\right\}_{(t=m=m+1, \ldots, m+n-1)}$. A cambio de esta prestación, el asegurado paga durante $k$ años, con $k \leq m$, una prima periódica, anticipada y constante de importe $P$ unidades monetarias.

La prima pura periódica de una operación de estas características, que simbolizamos como $P$, viene definida por la ecuación de equilibrio financiero-actuarial al principio de esta, esto es,

$$
P \cdot /{ }_{k} \ddot{a}_{x}=\sum_{t=m}^{m+n} \alpha_{t} \cdot(1+i)^{-t} \cdot\left(-y_{t} p_{x}\right.
$$

con,

$$
h_{k} \ddot{a}_{x}=\sum_{t=0}^{k}(1+i)^{-t} \cdot p_{x}
$$

de forma que el valor de la prima pura periódica es:

$$
P=\frac{\sum_{t=m}^{m+n} \alpha_{t}-(1+i)^{-t} \cdot t_{t} p_{x}}{\sum_{t=0}^{k}(1+i)^{-t} \cdot E_{x}}
$$

\subsubsection{Cálculo de la distribución de probabilidad de la rentabilidad real}

La rentabilidad anual de una renta de jubilación contratada a prima pura periódica es una variable aleatoria cuya distribución de probabilidad y el cálculo, mediante un proceso iterativo, de los valores correspondientes a cada una de las rentabilidades según el periodo considerado, viene dada por la siguiente expresión: 


\begin{tabular}{|c|c|c|}
\hline$\tilde{\imath}$ & Probabilidades & Cálculo \\
\hline$i_{0}$ & $/ m q_{x}$ & -1 \\
\hline$i_{1}$ & $:_{m / 1} q_{x}$ & $P \cdot \ddot{a}_{\bar{k} \mid i_{1}}=\alpha_{m} \cdot\left(1+i_{1}\right)^{-m}$ \\
\hline$i_{2}$ & $\vdots_{m+1 / 1} q_{x}$ & $P \cdot \ddot{a}_{\bar{k} \mid i_{2}}=\sum_{t=m}^{m+2} \alpha_{t} \cdot\left(1+i_{2}\right)^{-t}$ \\
\hline$i_{3}$ & $:_{m+2 / 1} q_{x}$ & $P \cdot \ddot{a}_{\bar{k} \mid i_{3}}=\sum_{t=m}^{m+3} \alpha_{t} \cdot\left(1+i_{3}\right)^{-t}$ \\
\hline$\vdots$ & $\vdots$ & $\vdots$ \\
\hline & ${ }_{m+n-2 / 1} q_{x}$ & $P \cdot \ddot{a}_{\bar{k} \mid i_{n-1}}=\sum_{t=m}^{m+n-1} \alpha_{t} \cdot\left(1+i_{n-1}\right)^{-t}$ \\
\hline$i$ & $:_{m+n-1} p_{x}$ & $P \cdot \ddot{a}_{\bar{k} \mid i_{n}}=\sum_{t=m}^{m+n} \alpha_{t} \cdot\left(1+i_{n}\right)^{-t}$ \\
\hline : & 1 & : \\
\hline
\end{tabular}

\subsubsection{Cálculo de la rentabilidad esperada}

La variable aleatoria valor actual del beneficio del producto, tiene la siguiente distribución de probabilidad:

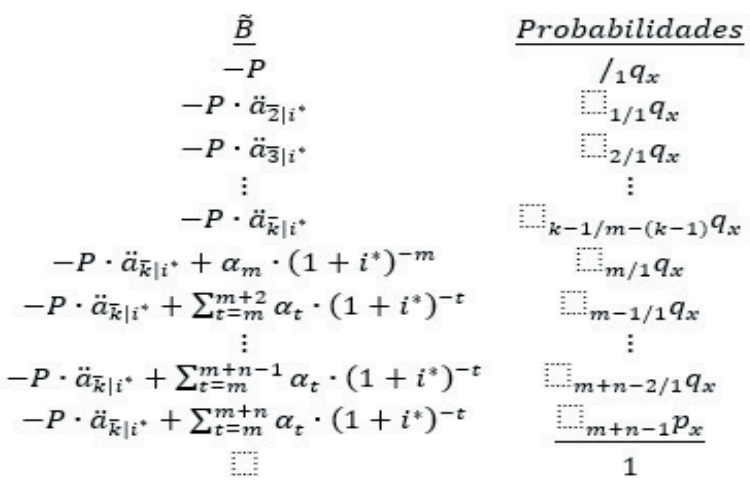

Para obtener $i^{*}$, calculamos $\mathbf{E}[\mathrm{B}]=\mathbf{O}$

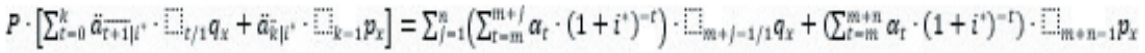

Y simplificando resulta:

$$
P \cdot\left[\sum_{t=0}^{k} \ddot{a}_{\overline{t+1} \mid i^{*}} \cdot \vdots_{t / 1} q_{x}+\ddot{a}_{\bar{k} \mid i^{*}} \cdot \vdots_{k-1} p_{x}\right]=\left(\sum_{t=m}^{m+n} \alpha_{t} \cdot\left(1+i^{*}\right)^{-t}\right) \cdot \vdots_{t} p_{x}
$$

Operando en el corchete de la ecuación (15), tenemos, 


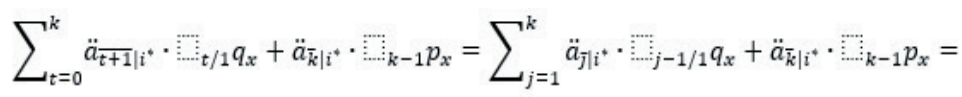

$$
\begin{aligned}
& =\sum_{j=1}^{k}\left(\sum_{t=0}^{j}\left(1+i^{*}\right)^{-t}\right) \cdot \square_{j-1 / 1} q_{x}+\sum_{t=0}^{k}\left(1+i^{*}\right)^{-t} \cdot{ }_{k-1} p_{x}= \\
& \left.=\sum_{t=0}^{k}\left(1+i^{*}\right)^{-t} \cdot \sum_{j=t}^{k}\right]_{j-1 / 1} q_{x}+\sum_{t=0}^{k}\left(1+i^{*}\right)^{-t} \cdot{ }_{k-1} p_{x}= \\
& =\sum_{t=0}^{k}\left(1+i^{*}\right)^{-t} \cdot\left({ }_{t} p_{x}-{ }_{k-1} p_{x}\right)+\sum_{t=0}^{k}\left(1+i^{*}\right)^{-t} \cdot{ }_{k-1} p_{x}= \\
& =\sum_{t=0}^{k}\left(1+i^{*}\right)^{-t} \cdot{ }_{t} p_{x}
\end{aligned}
$$

de forma que se obtiene el rendimiento esperado como solución de la ecuación,

$$
P \cdot \sum_{t=0}^{k}\left(1+i^{*}\right)^{-t} \cdot{ }_{t} p_{x}=\sum_{t=m}^{m+n} \alpha_{t} \cdot\left(1+i^{*}\right)^{-t} \cdot{ }_{t} p_{x}
$$

que coincide con la ecuación de equilibrio inicial que nos ha permitido obtener la prima pura periódica $P$. Esto significa que, cuando la renta de supervivencia se contrata a prima pura periódica, la rentabilidad esperada coincide con el tipo de interés técnico de la operación, esto es, $i^{*}=i$.

Resulta evidente que el resultado obtenido en (17) es idéntico al correspondiente al caso de prima pura única, simplemente substituyendo

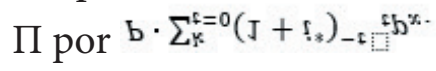

\section{APLICACIÓN NUMÉRICA}

Consideramos un asegurado de edad actual 40 años, que contrata una operación actuarial consistente en el pago de una unidad monetaria al inicio de la operación a cambio de recibir a partir de los 65 años, una renta de supervivencia prepagable y vitalicia, de cuantía constante igual a $\alpha$ unidades monetarias, para analizar el caso de la prima única y con pago de una unidad monetaria durante 10 años al inicio de cada periodo para estudiar el caso de la prima periódica. La operación, por tanto, está diferida 25 años, $m=25$, el tipo de interés técnico de la operación se 
establece en el 1,09\% o $i=0,0109$ y las tablas de mortalidad utilizadas para realizar los cálculos son las PASEM 2010.

Bajo estos supuestos, la rentabilidad real o tipo de interés efectivo anual de la operación de renta de supervivencia, en cada uno de los periodos de duración de esta, cuando la prima pagada, única y periódica, es pura, toma los siguientes valores:

\section{Tabla 1: Rentabilidad Real (tipo de interés efectivo anual). Renta de Su-} pervivencia Prima Pura Única (PPU) y Periódica (PPP)

\begin{tabular}{lllc} 
Edad & \multicolumn{1}{c}{ PPU } & \multicolumn{1}{c}{ PPP } & Probabilidad \\
65 & -1 & -1 & 0,134577129 \\
66 & $-0,08735106$ & $-0,110376105$ & 0,010993467 \\
67 & $-0,060488764$ & $-0,076643492$ & 0,012012423 \\
68 & $-0,04456592$ & $-0,056765833$ & 0,01319562 \\
69 & $-0,033337872$ & $-0,042805282$ & 0,014562786 \\
70 & $-0,024692561$ & $-0,032168959$ & 0,016135942 \\
71 & $-0,017769097$ & $-0,023708893$ & 0,017934818 \\
72 & $-0,012059303$ & $-0,016734167$ & 0,019986951 \\
73 & $-0,007199075$ & $-0,010889266$ & 0,022326678 \\
74 & $-0,003027176$ & $-0,005859442$ & 0,024978031 \\
75 & 0,000581744 & $-0,001599433$ & 0,027962636 \\
76 & 0,003754401 & 0,00219329 & 0,031276566 \\
77 & 0,006562737 & 0,005512622 & 0,034890668 \\
78 & 0,009067706 & 0,008468799 & 0,038731947 \\
79 & 0,011320877 & 0,011088632 & 0,042680498 \\
80 & 0,013349268 & 0,013418926 & 0,046540982 \\
81 & 0,015183484 & 0,015553396 & 0,047556282 \\
82 & 0,016857724 & 0,017473973 & 0,047994325 \\
83 & 0,01837082 & 0,019185745 & 0,047751686 \\
84 & 0,019755247 & 0,020797937 & 0,046765095 \\
85 & 0,021048298 & 0,022242865 & 0,04501643 \\
86 & 0,022170588 & 0,023573352 & 0,042529971 \\
87 & 0,023256368 & 0,024803837 & 0,039343881 \\
88 & 0,024255729 & 0,025886274 & 0,03553499 \\
89 & 0,025178115 & 0,026923111 & 0,031232853 \\
90 & 0,026031583 & 0,027872852 & 0,026625046 \\
91 & 0,026823048 & 0,028751082 & 0,021942632
\end{tabular}




$\begin{array}{llll}92 & 0,027558473 & 0,029564387 & 0,017429793 \\ 93 & 0,02826506 & 0,030318568 & 0,013312193 \\ 94 & 0,028883591 & 0,031018755 & 0,009756297 \\ 95 & 0,029479349 & 0,031669499 & 0,006849716 \\ 96 & 0,030053762 & 0,032274857 & 0,004600317 \\ 97 & 0,030546721 & 0,032838457 & 0,002951224 \\ 98 & 0,031046126 & 0,033372562 & 0,001805725 \\ 99 & 0,031506696 & 0,033881316 & 0,001051919 \\ 100 & 0,031940371 & 0,034345861 & 0,000582309 \\ 101 & 0,032349328 & 0,034781244 & 0,000305641 \\ 102 & 0,032735517 & 0,035189733 & 0,000160294 \\ 103 & 0,033100689 & 0,035573399 & 7,07 \mathrm{E}-05 \\ 104 & 0,033385384 & 0,035934134 & 2,85 \mathrm{E}-05 \\ 105 & 0,033713094 & 0,036235952 & 1,04 \mathrm{E}-05 \\ 106 & 0,034024071 & 0,036555205 & 3,38 \mathrm{E}-06 \\ 107 & 0,034307037 & 0,036855704 & 9,60 \mathrm{E}-07\end{array}$

Como se observa en la Tabla 1, la rentabilidad de la operación es negativa durante los 10 primeros años de cobro de los términos de la renta (desde los 65 años, en que el asegurado empieza a cobrar la renta, y hasta los 74 años) en el caso de que la prima pura pagada sea única. Cuando la prima pura es periódica, la rentabilidad de la operación es negativa durante los 11 primeros términos de la renta, desde los 65 y hasta los 75 años del asegurado. A partir de ese momento, la rentabilidad pasa a ser positiva y mayor cuantos más términos de la renta recibe el asegurado. El máximo valor de la rentabilidad se produce al término de la operación si el asegurado llega con vida a cumplir los 107 años en ambos casos.

El valor de la rentabilidad esperada, bajo los dos supuestos es $i^{*}=0,0109$, y coincide con el tipo de interés técnico de la operación.

Como coeficientes de riesgo consideraremos los dados por las siguientes dos probabilidades $p[\tilde{\imath} \geq 0]$ y $\mathrm{p}\left[\tilde{\imath} \geq i^{*}\right]$ e indican, respectivamente, la probabilidad de que el asegurado no tenga pérdidas con la operación y la confianza de dicho asegurado en obtener una rentabilidad real superior a la rentabilidad esperada. La Tabla (2) muestra los diferentes valores de los indicadores de riesgo para la renta de supervivencia en los escenarios de prima pura única y periódica: 


\section{Tabla 2: Indicadores de riesgo. Renta de Supervivencia a Prima Pura Única (PPU) y Periódica (PPP)}

$\begin{array}{lcc} & \text { PPU } & \text { PPP } \\ \text { Primer indicador de riesgo } & 0,71329587 & 0,68533323 \\ \text { Segundo indicador de riesgo } & 0,58043405 & 0,58043405\end{array}$

El primer coeficiente de riesgo es el resultado de sumar todas las probabilidades diferidas de fallecimiento desde el momento en que la rentabilidad deja de ser negativa para pasar a ser positiva, y hasta el final de la operación. Este indicador muestra la probabilidad de que la rentabilidad real sea positiva o nula y por tanto es mayor en el caso de la prima pura, puesto que el número de periodos con rentabilidad real negativa es menor. El segundo indicador de riesgo, que muestra la confianza del asegurado en obtener un tipo de interés real mayor o igual a la rentabilidad esperada, se obtiene sumando las probabilidades diferidas de fallecimiento a partir del pago del término de la renta, en el que el tipo de interés obtenido supera por primera vez al tipo de interés esperado, $\mathrm{y}$ hasta el final de la operación. Este segundo coeficiente de riesgo es el mismo en los dos escenarios analizados puesto que la rentabilidad esperada en ambos casos coincide con el tipo de interés técnico de la operación.

\section{CONCLUSIONES}

Desde el año 2015 las compañías de seguros tienen la obligación de informar a sus asegurados de la rentabilidad que pueden obtener al contratar una determinada operación de seguros de vida, en la modalidad de ahorro

Como primera conclusión del trabajo, destacamos la importancia del análisis estocástico de cualquier operación actuarial y en particular de las rentas de supervivencia sobre una persona. Determinando la distribución de probabilidad de la variable aleatoria tanto efectivo de rentabilidad y 
de la correspondiente al valor actual de la función beneficio neto del producto obtenemos los instrumentos adecuados para llevar a cabo el análisis del riesgo de las mismas.

De la distribución de esta última variable aleatoria, obtenemos el tanto efectivo de rentabilidad esperada, igualando a cero su valor esperado. Pero este valor esperado, no deja de ser un promedio y su representatividad puede ser muy pequeña cuando queremos analizar de forma individual la operación para un asegurado, de forma similar a lo que sucede cuando asociamos la renta per cápita como renta media de la población, a uno de sus integrantes de forma individual. Como consecuencia de ello, para tomar decisiones sobre una operación actuarial, no podemos utilizar simplemente el tanto efectivo de rentabilidad esperada, como sucedía con el tanto efectivo anual equivalente en las operaciones financieras, sino que debemos añadir información adicional sobre el riesgo de la operación cuantificando la confianza que puede tener el asegurado en alcanzar la rentabilidad esperada, así como de obtener una rentabilidad negativa.

\section{REFERENCIAS BIBLIOGRÁFICAS}

Devesa Carpio, J. E., Devesa Carpio, M., Alonso Fernández, J. J., Domínguez Fabián, I., Meneu Gaya, R. \& Encinas Goenechea, B. (2016). El reto de las entidades aseguradoras ante la introducción de la rentabilidad esperada en España. Universia Business Review, 52, 168-197.

Moreno Ruiz, R., Trigo Martínez, E., Gómez Pérez-Cacho, O. \& Escobar López, R. N. (2017). Rentabilidad esperada en seguros de vida: análisis actuarial de la metodología de cálculo a la luz de la Orden ECC/2329/2014, de 12 de diciembre. Anales del Instituto de Actuarios Españoles, $4^{a}$ época, 23, $102-128$.

Devesa Carpio, J.E., Devesa Carpio, M., Alonso Fernández, J.J., Domínguez Fabián, I., Encinas Goenechea, B. \& Meneu Gaya, R. (2013). “La rentabilidad actuarial como método de comparación de las operaciones financieras y aseguradoras”. En E. Gómez, M. Guillén y F. Vázquez (eds.): 
Investigaciones en Seguros y Gestión de Riesgos: Riesgo 2013. Madrid, Fundación Mapfre, pp. 85-98.

Orden ECC/2329/2014, de 12 de diciembre, por la que se regula el cálculo de la rentabilidad esperada de las operaciones de seguro de vida. Ministerio de Economía y Competitividad, 2014. Recuperado de https://www.boe.es/ buscar/doc.php?id=BOE-A-2014-12974.

Pérez-Fructuoso, M.J. y Alegre Escolano, A. (2019). Cálculo estocástico de la rentabilidad financiero-fiscal de una operación de capital al final del periodo de fallecimiento del asegurado. Revista de Investigación Operacional, vol. 40, n 4, 475-495.

Pérez-Fructuoso, M.J. y Alegre Escolano, A. (2018). Cálculo de la rentabilidad esperada y cuantificación del riesgo en una operación de ahorro de capital diferido a prima (pura y comercial) única. Rect@, vol 19, n 1, 17-34.

Promislow, S.D. (2015). Fundamentals of Actuarial Mathematics, 3rd Edition, Ringgold Inc, Beaverton.

Real Decreto 1060/2015, de 20 de noviembre, de ordenación, supervisión y solvencia de las entidades aseguradoras y reaseguradoras. Recuperado de https://www.boe.es/buscar/doc.php?id=BOE-A-2015-13057. 